\title{
Medium-Term Effects of Bisoprolol Administration on Renal Hemodynamics and Function in Mild to Moderate Essential Hypertension
}

Salvatore Paterna, Gaspare Parrinello, Pietro Di Pasquale, Daniele Torres, Gabriella La Rocca, Roberta Antona, Laura Vernuccio, Ersilia Fornaciari, Antonina Tarantino, Ercole Piccione, Sergio Fasullo, Giuseppe Licota

This article, appearing in the November/December 2007 edition of Advances in Therapy (volume 24, Issue 6, pages 1260-1270) was funded by a grant from Bayer Schering Pharma. 